\title{
Silicon Lens Optimization to Create Diffuse, Uniform Illumination from Incoherent THz Source Arrays
}

\author{
Robin Zatta ${ }^{1}$ (D) . Daniel Headland ${ }^{2}$ - Eamal Ashna ${ }^{1} \cdot$ Ritesh Jain $^{3}$. \\ Philipp Hillger ${ }^{1}$ • Janusz Grzyb ${ }^{1}$ • Ullrich R. Pfeiffer ${ }^{1}$
}

Received: 28 August 2021 / Accepted: 14 October 2021 / Published online: 26 October 2021

(C) The Author(s) 2021

\begin{abstract}
Arrays of terahertz $(\mathrm{THz})$ sources provide a pathway to overcoming the radiation power limitations of single sources. Several independent sources of $\mathrm{THz}$ radiation may be implemented in a single integrated circuit, thereby realizing a monolithic $\mathrm{THz}$ source array of high output power. Integrated $\mathrm{THz}$ sources must generally be backside-coupled to extended hemispherical dielectric lenses in order to suppress substrate modes and extract $\mathrm{THz}$ power. However, this lens also increases antenna gain and thereby produces several non-overlapping beams. This is because individual source pixels are relatively large. Hence, their spatial separation on-chip translates to angular separation in the far-field. In other words, there are gaps in their field of view into which very little $\mathrm{THz}$ power is projected. Therefore, they cannot homogeneously illuminate an imaging target. This article presents a simple, practical, and scalable method to convert arrays of incoherent $\mathrm{THz}$ sources into a diffuse, uniform illumination source without the need for reducing pixel size. Briefly, individual beam divergence is optimized by tailoring the dimensions of the extended hemispherical dielectric lens such that the far-field beams of adjacent source pixels overlap and combine to form a uniform far-field beam. We applied this method to an incoherent $8 \times 8$-pixel THz source array radiating $10.3 \mathrm{dBm}$ at $0.42 \mathrm{THz}$ as a proof of concept and thereby realized a $10.3-\mathrm{dBm} 0.42-\mathrm{THz}$ diffuse, uniform illumination source that was then deployed in a demonstration of $\mathrm{THz}$ active imaging.
\end{abstract}

Robin Zatta

zatta@uni-wuppertal.de

1 University of Wuppertal, 42119, Wuppertal, Germany

2 Terahertz Engineering Laboratory, School of Electrical and Electronic Engineering, The University of Adelaide, South Australia, 5005, Australia

3 Astrome, Bengaluru, India 
Keywords $\mathrm{THz}$ active imaging $\cdot \mathrm{THz}$ source array $\cdot \mathrm{THz}$ uniform illumination source $\cdot$ Incoherent power-combining $\cdot$ Extended hemispherical lens

\section{Introduction}

High-power, uniform-profile illumination sources operating at high frequencies are a much-needed but missing element in camera-based terahertz (THz) active imaging applications to produce high-quality images with a high, homogeneously distributed signal-to-noise ratio (SNR) and high spatial resolution. However, the radiation power of individual $\mathrm{THz}$ sources decreases drastically with respect to increasing frequency $[1,2]$. To address this, several sources may be operated together to realize source arrays of greater overall radiation power. In this regard, silicon-integrated technologies are viable as they offer a high fabrication yield to incorporate such arrays into a single chip. In recent years, both coherent [3-10] and incoherent [1115] single-chip $\mathrm{THz}$ source arrays have been reported. A salient difference between these two approaches is that coherent source arrays require an on-chip synchronization mechanism for phase-locking. Although this may incur some restrictions upon performance and array size, recent works have produced highly scalable devices [6, 9]. Despite ongoing advances in efficient on-chip synchronization methods, incoherent devices remain more scalable and produce higher radiation power [12]. Aside from array size and radiation power, there are other crucial differences between coherent and incoherent approaches. Coherent radiation can prove detrimental to image fidelity in many situations, as speckles form due to wave interference [16, 17], which is not the case with incoherent radiation [13]. Furthermore, unlike communications and radar, amplitude-only $\mathrm{THz}$ imaging does not require a coherent signal. For these reasons, the objective of this work is to explore incoherent $\mathrm{THz}$ source arrays for active imaging applications. A uniform-amplitude beam profile is desired in order that an imaging target is homogeneously illuminated, yielding constant dynamic range across the imaging plane. However, in contrast to this aim, contemporary incoherent $\mathrm{THz}$ source arrays produce several non-overlapping beams $[12,13]$, which produces dead zones in the imaging plane, as shown in Fig. 1(a). These dead zones have two causes: the source array pixels are large relative to a wavelength, and pixel pitch translates into an angular separation of free-space beams. Thus, according to [18], overlapping beams can only be achieved with a lens-coupled source array if the pixel cells are small enough so that they can be close together. However, the size of source pixels cannot be easily reduced due to the required power generation networks. Therefore, another solution is required to increase the beam overlap through an optimized silicon lens, yielding a uniform beam, as shown in Fig. 1(b).

In the past, significant research effort has been dedicated to the maximization of directivity for a single radiating element coupled to an extended hemispherical silicon lens as well as associated problems [19-26]. It has also been shown that beams from multiple small coherent sources incorporated into a single chip can be combined into a single Gaussian beam [27]. However, creating diffuse, uniform illumination from large-scale arrays of incoherent $\mathrm{THz}$ sources has not yet been demonstrated. 


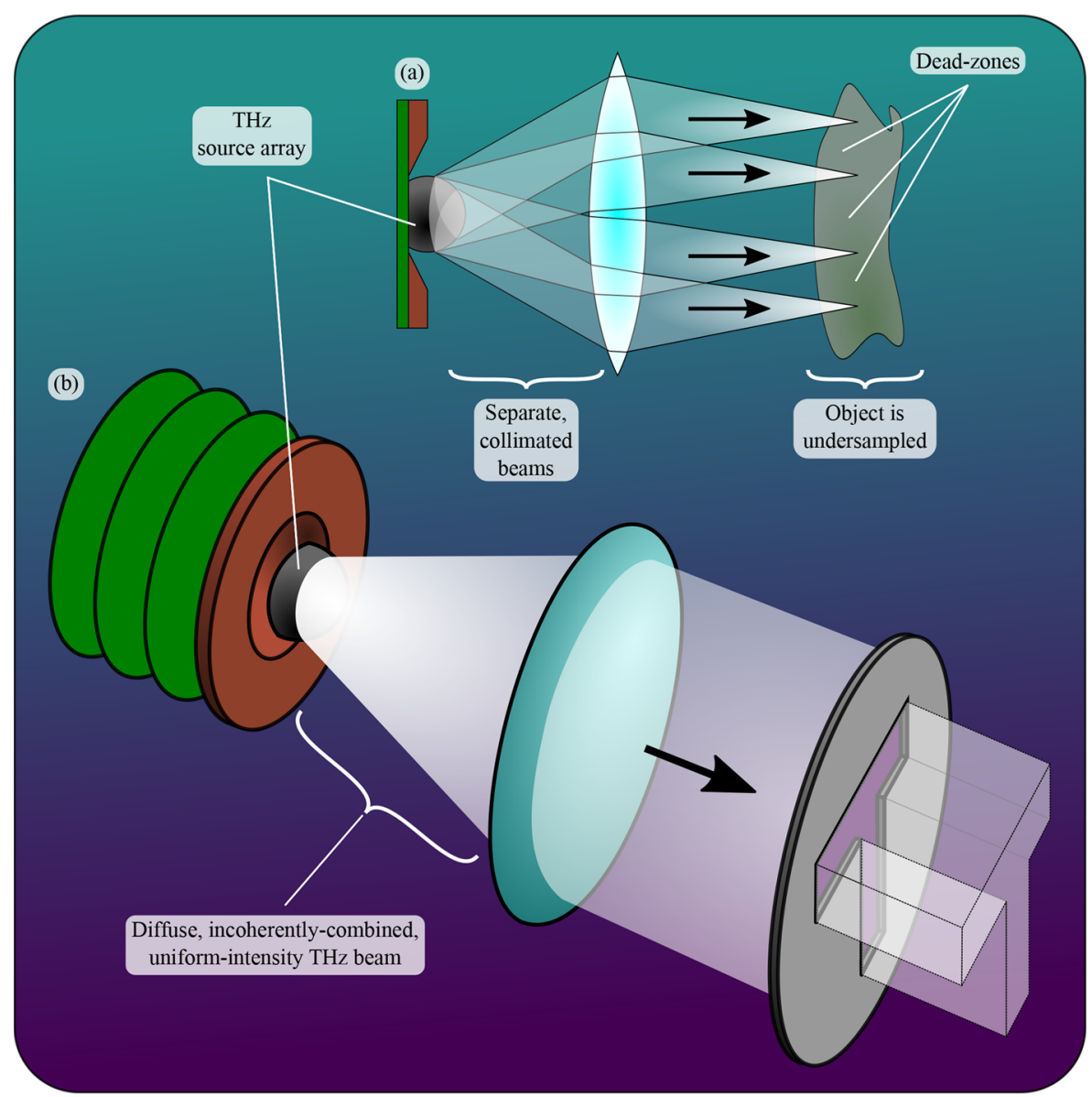

Fig. 1 Illustration of the primary motivation of this study, showing (a) how previous high-power $\mathrm{THz}$ incoherent source arrays $[12,13]$ produce directional, non-overlapping beams that lead to undersampling of the image plane, and (b) the primary objective of this study; a uniform-intensity, high-power $\mathrm{THz}$ illumination source

To this end, this article presents a method to convert an arbitrary array of $\mathrm{THz}$ sources into a diffuse, uniform illumination source by tailoring the geometry of the backside-coupled lens - without the need for any modification to the integrated circuit itself-and a proof of concept is realized using a $0.42-\mathrm{THz}$ device with a power of $10.3 \mathrm{dBm}$. Silicon-only single-shot $\mathrm{THz}$ active imaging is demonstrated together with a CMOS camera based on the focal plane array (FPA) from [28]. Previous attempts at incoherent power-combining of $\mathrm{THz}$ source arrays in free space have relied upon multi-device scaling [29] or lossy external optics [30]. Both of these approaches are bulky and inefficient. In comparison, the approach taken here is a single-chip-to-single-lens solution. It exhibits significant advantages in terms of both compactness and efficiency. 


\section{THz Source Array}

The following is a summary of the details of the incoherent silicon-integrated source array chip used here, which has been presented in detail in [12]. For more details, the reader is referred to the cited work.

The chip is implemented in a commercial SG13G2 SiGe BiCMOS process available from IHP microelectronics, with $350-\mathrm{GHz} / 450-\mathrm{GHz} f_{t} / f_{\max }$ SiGe HBT transistors. This chip incorporates $8 \times 8$ incoherent $\mathrm{THz}$ sources. Together, they radiate a rotal radiation power of $10.3 \mathrm{dBm}$ at $\sim 0.42 \mathrm{THz}$. Each source pixel is composed of a power generation network coupled to an on-chip circular slot antenna. Each source pixel cell, thereby, occupies a die area of $365 \mu \mathrm{m} \times 365 \mu \mathrm{m}$. For THzrange power generation, free-running fundamental Colpitts oscillators, followed by a common-collector doubler, are used. The oscillators are not mutually phase-locked. Consequently, the radiation frequencies of the source pixels are not the same, making the source array an incoherent device. The difference in the radiation frequency has two causes: process variations and variations in bias. Related to this, mutual pixel coupling, which can cause two or more pixels to lock onto the same oscillation frequency, is mitigated by placing grounding shields around the source pixels and the silicon lens. Grounding shields prevent any leakage from electromagnetic fields, whereas the lens suppresses undesired substrate modes [31] by acting as a semi-infinite substrate. According to [32], an array atop a silicon lens is subject to a mutual pixel coupling on the order of -25 to $-30 \mathrm{~dB}$. In the source array, the isolation is further enhanced by the architecture of the source pixels. In particular, the frequency doubler after the oscillator prevents any out- and in-coupling of the fundamental oscillator signal. The lens not only suppresses unwanted substrate modes but also improves the front-to-back radiation ratio and imparts mechanical rigidity and thermal stability to the silicon chip [22]. For these reasons, the vast majority of integrated $\mathrm{THz}$ sources are coupled to extended hemispherical silicon lenses [2, 33-35].

\section{Lens Design}

\subsection{Simulation Model}

For pre-estimation of the optimal lens extension length required for a minimum -3-dB beam overlap among all source pixel pairs and to create a uniform beam, numerical simulations were utilized based on the theory from [22, 23]. It is noted that full-wave simulations are impractical because the silicon lens is electrically large. Our simulation model accounts for Fresnel losses, which is to say, the amplitude pattern of fields on the external surface of the lens accounts for reflection losses. These losses are spatially dependent, and hence reflection impacts the overall far-field radiation pattern and directivity. Aside from Fresnel losses, other reflection-related effects are not modeled; specifically, the fields that are back-reflected into the lens volume and undergo multiple subsequent reflections before ultimately being radiated via the lens surface. Nevertheless, multiply reflected radiation is anticipated to contribute 


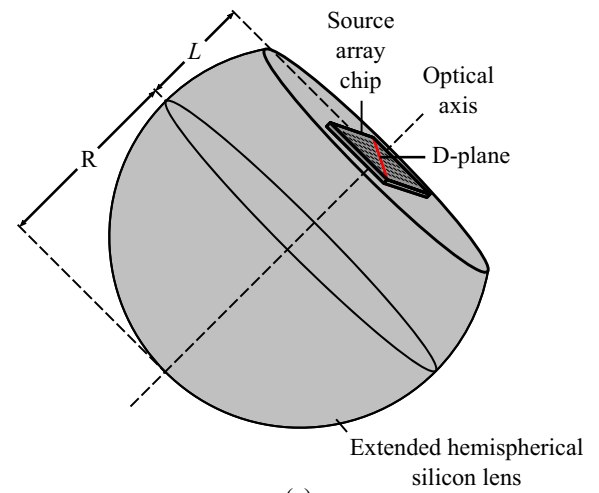

(a)

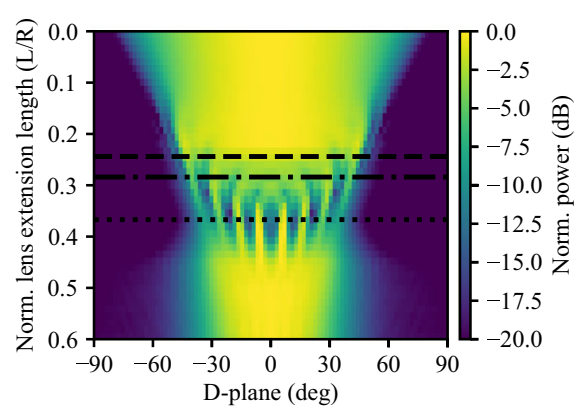

(b)

Fig. 2 (a) Illustration of the chip-to-lens assembly, indicating the lens geometrical parameters $L$ and $R$. (b) Pre-estimation simulation results, showing combined D-plane cuts, as indicated in (a), for normalized lens extension length of $0-0.6$. The horizontal lines refer to values selected for experimental characterization

primarily to sidelobes, and hence it is of little interest to this work, as we aim to produce overlap between main lobes. The main difference to the math provided in the cited works is the radiation pattern of the lens-internal antenna. We employ a more abstract beam pattern in the interest of generality:

$$
\left|\mathbf{E}_{\text {Source }}\left(\theta^{\prime}, \phi^{\prime}\right)\right|=\left(\frac{\cos \theta^{\prime}+1}{2}\right)^{q},
$$

where the value $q$ makes it possible to set any desired internal beam divergence,

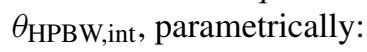

$$
q=\log \left(\frac{1}{2}\right) \cdot\left(\frac{2 \cdot \log \left(\frac{\cos \theta_{\mathrm{HPBW}, \text { int }}}{2}\right)}{2}\right)^{-1} .
$$

\subsection{Simulation Results}

In order to obtain a comprehensive picture of achievable far-field radiation patterns, combined far-field radiation patterns were determined for a range of normalized lens extension lengths from $L / R=0.0$ to $L / R=0.6$, where $L$ is lens extension length, and $R$ is lens radius, as shown in Fig. 2(a). To this end, single-pixel far-field radiation patterns were computed for all $8 \times 8$ source pixels located at the base of a $15-\mathrm{mm}$ (71.7 $\lambda$ ) diameter extended hemispherical silicon lens. The summation of these singlepixel beams then produced the combined field radiation patterns. The unnormalized power was used in this case to account for differences in directivity. The permittivity of the silicon lens was set to $11.67^{1}$. It is noted that a precise permittivity value is critical to avoid a systematic offset in the lens extension length.

\footnotetext{
${ }^{1}$ http://www.tydexoptics.com/products/thz_optics/thz_materials/
} 
The intended comprehensive picture can be transported best through cuts along the diagonals of the combined far-field radiation patterns (D-plane cuts) as a function of the normalized lens extension length, shown in Fig. 2(b). As seen from this figure, the D-plane is oversampled for small values of the lens extension length. The same applies to large values, while medium values create gaps in the field of view (FoV) into which very little power is projected. The latter case corresponds to a lens extension that is optimized for maximum directivity. It is also observed that small values of lens extension length allow the generation of uniform or flat-top illumination, as the edges of the combined far-field radiation pattern are sharply separated. In contrast, large values smear the edges. Thus, large extensions have the effect of producing a more Gaussian illumination. The cause for the differences in the beam shape generated is because a hemispherical "focal surface" with inverted orientation to the hemispherical surface of the lens exists inside the dielectric, with a different far-field direction associated with each point on this surface. In normal operation, the lowest point of this focal surface corresponds to the lens extension. To be appropriately focused, off-axis pixels would have to be shifted upward in the positive $z$-direction to compensate for the curvature of the focal surface. If we either shorten or increase the lens extension, the directivity of the center pixel is reduced because it is essentially defocused. When the lens extension is increased, the directivity of the off-axis pixels is reduced more than that of the center pixel because the off-axis pixels are farther away from the focal surface. On the other hand, when shortened, the off-axis pixels are raised in the positive $z$-direction. Thus, they are closer to the focal surface than the center pixel. Therefore, the reduction in directivity is less than the directivity reduction for center pixels. Ultimately, the desired beamform determines whether the lens extension needs to be increased or shortened, with the former yielding a Gaussian beam and the latter a uniform beam. Another noteworthy point observable from the simulated D-plane cuts is that the FoV decreases with increasing lens extension length. A small FoV is desirable concerning realizing small $f$-number quasi-optical systems for $\mathrm{THz}$ active imaging that are easier to align than those with large $f$-numbers. Since the overarching aim was to create uniform illumination from the source array at a minimal FoV, a lens extension length just above the intermediate position that causes gaps in the FoV is the best option. Here, uniform illumination is produced at a minimal FoV.

Following the above discussion, the normalized lens extension length of 0.244 was selected for fabrication and testing to create uniform illumination from the source array, as indicated by the dashed line. In addition, two other cases with normalized lens extension lengths of 0.284 and 0.367 were selected for fabrication and testing in the interest of providing a more complete and comprehensive picture. The first case $(0.284)$ represents the aplanatic case for silicon, which is associated with zero coma and zero spherical aberration for a central pixel [19]. This first case is intended to fill some gaps in the FoV. The second case (0.367) is intended to undersample the FoV due to non-overlapping beams. This second case refers to the lens design implemented in the previous works $[12,13]$. 


\section{SiGe HBT source array}

(10.3 $\mathrm{dBm}$ at $0.42 \mathrm{THz}$; $-3.54 \mathrm{dBm}$ per source pixel )

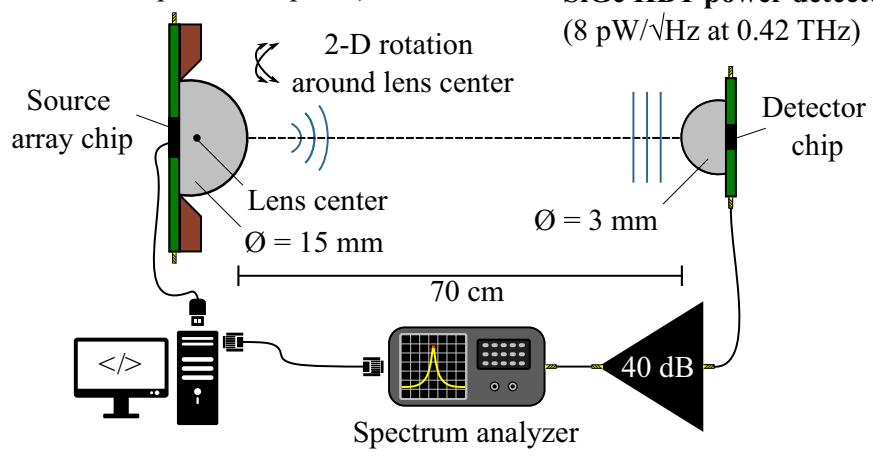

Fig. 3 Illustration of the experimental setup employed for far-field radiation pattern measurements

\section{Characterization}

\subsection{Experimental Setup}

Figure 3 shows the experimental setup employed for far-field radiation pattern characterization. For relative power measurements, the radiated $\mathrm{THz}$ signal of the source arrays with different lens extension lengths was successively collected with a SiGe HBT THz direct power detector with $700-\mathrm{V} / \mathrm{W}$ responsivity and 8-pW/ $\sqrt{\mathrm{Hz}} \mathrm{NEP}$ at around $0.42 \mathrm{THz}$ [36]. A far-field distance separated the source arrays and the detector. Specifically, the distance was $70 \mathrm{~cm}$, which is above the Fraunhofer distance of $2 D^{2} / \lambda=63 \mathrm{~cm}$ for a lens diameter of $15 \mathrm{~mm}$ and the source array radiation frequency of $\sim 0.42 \mathrm{THz}$. The detector was fixed in place and connected to a spectrum analyzer via a $40-\mathrm{dB}$ voltage amplifier. The source arrays were mounted onto a sixaxis table-top robot arm in order to facilitate a rotational raster scan. Each source array was scanned over a $\pm 36^{\circ} \times \pm 36^{\circ}$-sector of the hemisphere to capture far-field beams of all source pixels.

\subsection{Measured Results}

Figure 4(a), (b), and (c) show the measured full far-field radiation patterns with normalized power for normalized lens extension lengths of $0.367,0.284$, and 0.244, respectively. Corresponding D-plane cross-section cuts are compared to simulation in Fig 4(d)-(f). It can be seen from these results that the beam overlap (or fill factor) indeed increases with decreasing lens extension length, as intended. The source arrays coupled to extended hemispherical silicon lenses of $0.367,0.284$, and 0.244 normalized lens extension length cover experimentally tested $\mathrm{FoVs}$ of $50^{\circ}, 55^{\circ}$, and $55^{\circ}$, with $3-\mathrm{dB}$ fill factors of $6.5 \%, 53.4 \%$, and $99.98 \%$, respectively. The corresponding peak-to-peak ripple values are $\sim 20 \mathrm{~dB}, 8.6 \mathrm{~dB}$, and $3.5 \mathrm{~dB}$. Thus, the 


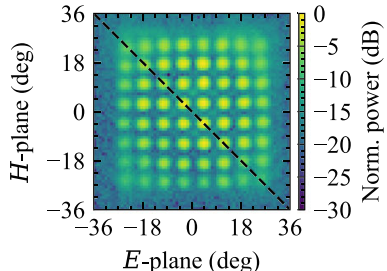

(a)

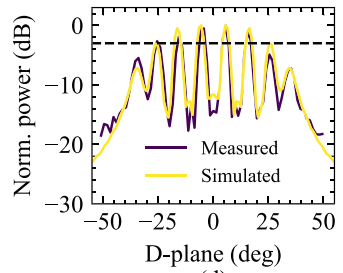

(d)

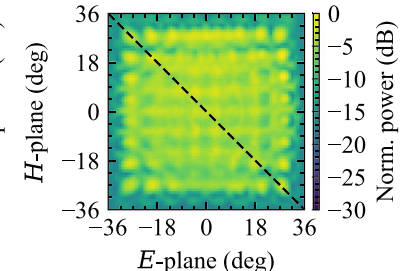

(b)

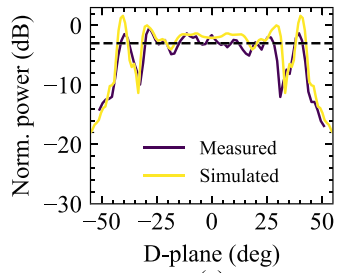

(e)

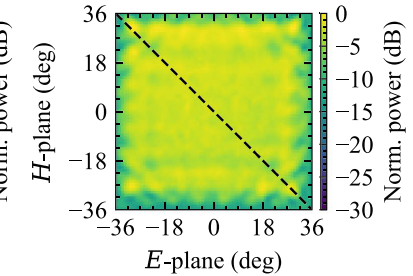

(c)

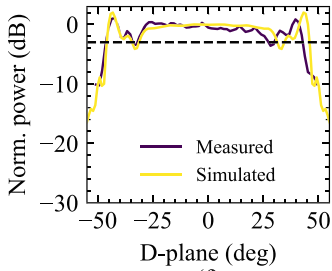

(f)

Fig. 4 Measured combined far-field radiation patterns of source arrays using 15-mm diameter silicon lenses with different normalized lens extension lengths of 0.367 (a), 0.284 (b), and 0.244 (c) and directivity. In (d)-(f), D-plane cuts along the dashed lines in the corresponding complete far-field radiation patterns from (a)-(c) are compared with the results obtained from simulations. In all subfigures, normalization is performed with respect to the maximum power within the constant radius FoV

primary objective of this study, namely the realization of a high-power, diffuse, uniform-intensity $\mathrm{THz}$ source, has been achieved. Finally, given that the measured combined far-field radiation patterns are in excellent agreement with their simulated counterparts, we may conclude that the simulation model has been successfully validated.

\section{Demonstration of Silicon-Only THz Active Imaging}

\subsection{Single-Shot Imaging Setup}

An illustration and photograph of the experimental setup are shown in Fig. 5(a) and (b), respectively, with which silicon-only $\mathrm{THz}$ active imaging was performed. That is to say, both the source and detector of $\mathrm{THz}$ waves are silicon-based integrated circuits. The two source arrays coupled to lenses of the highest and lowest lens extension length were successively deployed in a collimated beam setup that consists of a series of optics and a commercially available CMOS $\mathrm{THz}$ camera from Ticwave $\mathrm{GmbH}$, Wuppertal, Germany ${ }^{2}$. This camera is based on the FPA from [28]. It is noted that a variety of $\mathrm{THz}$ applications have been demonstrated with this camera ranging from shadow imaging [28] over light-field imaging [37-39] to source characterization [4043], among others. The total radiation power of the source array is $10.3 \mathrm{dBm}$ [12], and the video-rate camera NEP at the source array radiation frequency of $0.42 \mathrm{THz}$ is

\footnotetext{
${ }^{2}$ https://ticwave.com/index.php/shop/ticmos-1kpx
} 

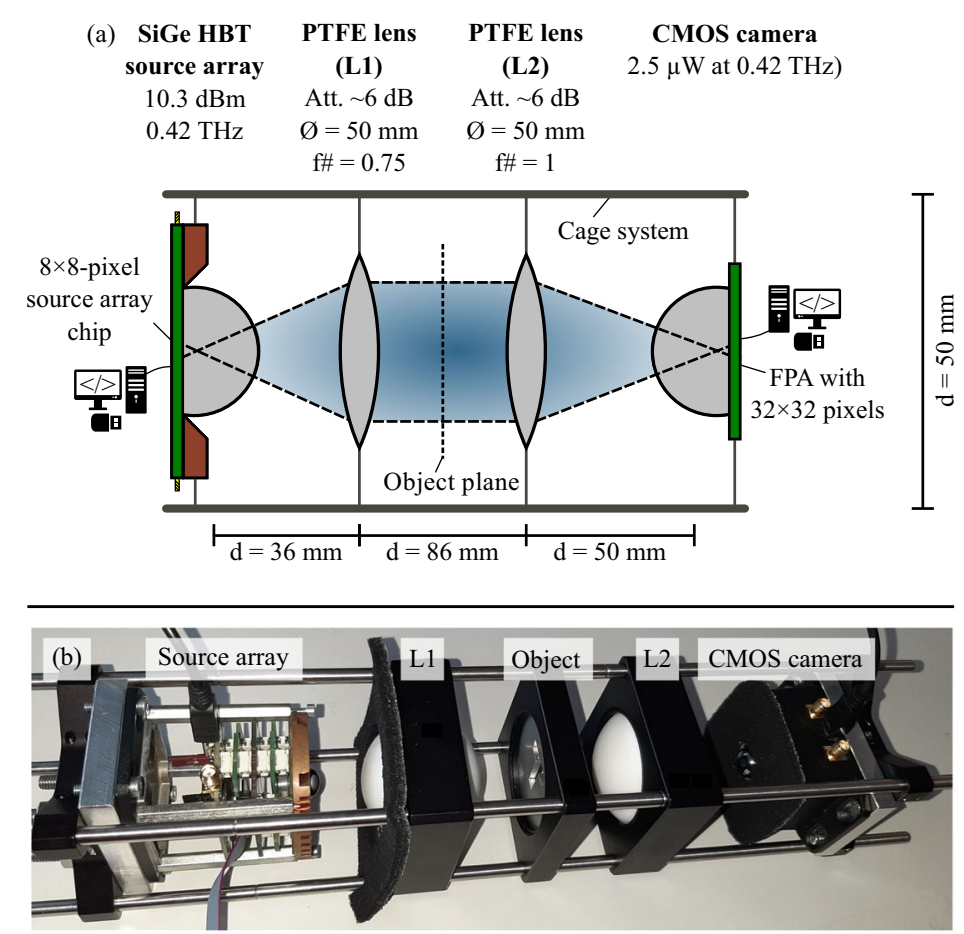

Fig. 5 Illustration of the experimental setup employed for and silicon-only $\mathrm{THz}$ active imaging (a), and a photo of that (b)

$2.5 \mu \mathrm{W}$ [44]. All components were fixed within a cage system to ensure mechanical rigidity. The source arrays and the camera are operated via USB. The presented imaging system is portable, spanning an overall system size of $136 \mathrm{~mm} \times 50 \mathrm{~mm} \times 50 \mathrm{~mm}$. The key enabling factor in implementing this compact, portable $\mathrm{THz}$ active imaging system is the incoherent operation of the source array and camera. It is noted that the proposed $\mathrm{THz}$ uniform illumination source array-based active imaging setup is not tied to a CMOS camera. Other direct power detectors, such as microbolometers [45], CMOS-NEMS [46], SiGe HBTs [47], and Schottky barrier diodes [48], can replace the CMOS detectors, provided that they can be integrated into a single chip.

The beam propagation within the imaging setup works as follows. The optical train consists of two PTFE lenses. At the source array side, a 50-mm diameter collimating PTFE lens with an f-number of 0.75 (L1), which closely corresponds to the source array FoVs of $50^{\circ}$ and $55^{\circ}$, is employed for collimation. The source array lens center, where central rays of the source pixel beams cross [37], coincides with the focal point of L1. The source array emits diverging wavefronts emanating from the focal point of L1. Consequently, collimated waves are provided in the object plane, where they illuminate an imaging target. The camera lens center, where central rays of camera pixel beams cross [37], coincides with the focal point of the right collimating PTFE lens (L2; diameter of $50 \mathrm{~mm}$ and f-number of 1), which closely corresponds to the $46^{\circ} \mathrm{FoV}$ of the camera. Thus, the object plane is projected onto the camera lens. 


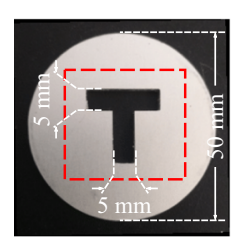

(a)

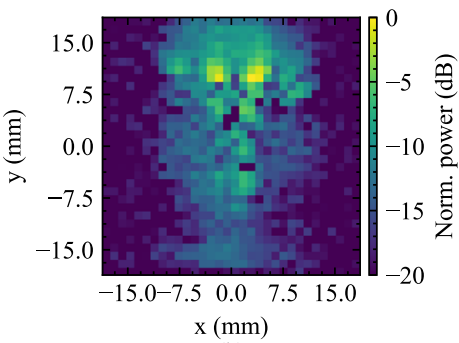

(b)

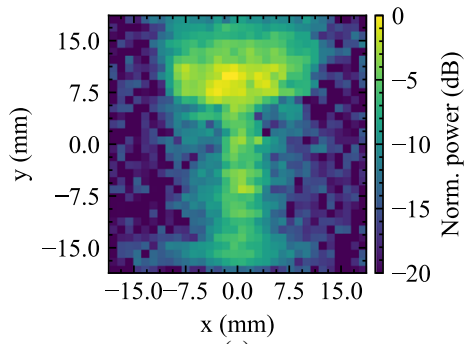

(c)

Fig. 6 Object (a) imaged at $0.42 \mathrm{THz}$ with the CMOS THz camera using 2.75-mm (b) and 1.83-mm (c) lens extension length-coupled source array. The " $\mathrm{T}$ " is only clearly visible in the oversampled THz image in (c)

Ultimately, an object under illumination may appear as an inverted image on the FPA surface. Since the pixel beams of the source array and the pixel beams of the camera are collimated (or planarized) in the object plane, far-field conditions prevail exactly at this point. Moving away from this position will cause an object to be out of focus. Such defocusing will cause producing blurred images.

\subsection{Imaging Results}

A 3-mm thick, 50-mm diameter metal plate with a T-shaped cut-out served as the imaging object, as shown in Fig. 6(a). This object blocked most of the beam, allowing only a small portion of $\mathrm{THz}$ power to pass. Figure 6(b) and (c) show $\mathrm{THz}$ images of the marked portion of the object acquired with the camera using the $2.75-\mathrm{mm}$ and 1.83-mm extended hemispherical silicon lens-coupled source array, respectively. It can be seen that the $\mathrm{THz}$ image depicted in Fig. 6(b) is undersampled, whereas the one depicted in Fig. 6(c) is oversampled. Hence, the " $T$ " is only recognizable in the latter. Raw images without any image-processing applied are shown. Each image was acquired over a $\sim 30$-second time span, as the CMOS camera was operated at $30 \mathrm{fps}$ and averaged 1024 frames. Frame-averaging was applied to increase the SNR [49]. Diffraction at the internal edges of the "T" may play a role in the blurring observed at the internal object edges, which results in the degradation of overall image quality. It should be noted that this diffraction-induced degradation of the overall image quality is not due to the imaging system itself but to the tiny slit width of the imaging object, which is close to a wavelength of just under $1 \mathrm{~mm}$.

\section{Conclusion}

In this article, a practical and scalable method to operate a single-chip array of incoherent $\mathrm{THz}$ sources as a uniform illumination source has been demonstrated. For demonstration, this method was applied to the source array from [12], thereby realizing a $0.42-\mathrm{THz}$ source that distributes $10.3 \mathrm{dBm}$ evenly over its FoV. Briefly, our 
method is based upon optimization of the geometry of a backside-coupled silicon lens. Silicon-only $\mathrm{THz}$ active imaging has been successfully demonstrated together with a CMOS THz camera based on the FPA presented in [28].

All single-element integrated $\mathrm{THz}$ sources suffer from a low radiation power and are relatively large in terms of wavelength [1,2]. Furthermore, lens coupling is ubiquitous among THz sources [2, 33-35, 50]. For these reasons, the presented technique of reduced-extension length hemispherical silicon lens-coupled $\mathrm{THz}$ source arrays is of general utility as a free-space power-combining technique; for example, also for incoherent source arrays of resonant tunneling diodes [14], or photomixers [15]. Furthermore, when the source that constitutes the array is mutually phase-locked, this would naturally yield coherent power-combining. This manner of scalable coherent power-combining could be essential to photo-mixing emitters, which exhibit broad frequency tunability, but currently suffer from a low radiation power at high frequencies [27, 34, 35]. An array of photo-mixing sources could be fed from a single high-power beating laser, thereby leading to innate synchronization among adjacent sources.

Acknowledgements We gratefully acknowledge Ticwave GmbH, Wuppertal, Germany, for providing the CMOS THz camera.

Funding Open Access funding enabled and organized by Projekt DEAL. This work was supported by the German Research Foundation (Deutsche Forschungsgemeinschaft, DFG) under the individual grant projects "Silicon Integrated Smart Terahertz Source Arrays for Reciprocal Imaging (SI-STAR)" with grant number DFG PF 661/16-1 and "Spatially-Mapped Mobile Terahertz Spectroscopy (T-MAP)" with grant number DFG PF 661/18-1. Furthermore, this work was supported by the European Research Council (ERC) under "Direct Temporal Synthesis of Terahertz Light Fields Enabling Novel Computational Imaging (DIRECTS)" with grant number ERC-2020-ADG 101019972.

Open Access This article is licensed under a Creative Commons Attribution 4.0 International License, which permits use, sharing, adaptation, distribution and reproduction in any medium or format, as long as you give appropriate credit to the original author(s) and the source, provide a link to the Creative Commons licence, and indicate if changes were made. The images or other third party material in this article are included in the article's Creative Commons licence, unless indicated otherwise in a credit line to the material. If material is not included in the article's Creative Commons licence and your intended use is not permitted by statutory regulation or exceeds the permitted use, you will need to obtain permission directly from the copyright holder. To view a copy of this licence, visit http://creativecommons.org/licenses/by/4.0/.

\section{References}

1. C.M. Armstrong, IEEE Spectr. 49(9), 36 (2012). https://doi.org/10.1109/MSPEC.2012.6281131.

2. P. Hillger, J. Grzyb, R. Jain, U.R. Pfeiffer, IEEE Trans. THz Sci. Technol. 9(1), 1 (2019).

3. R. Han, C. Jiang, A. Mostajeran, M. Emadi, H. Aghasi, H. Sherry, A. Cathelin, E. Afshari, IEEE J. Solid-State Circuits 50(12), 2935 (2015). https://doi.org/10.1109/JSSC.2015.2471847.

4. Y. Tousi, E. Afshari, in Proc. IEEE Int. Solid-State Circ. Conf. Dig. Techn. Papers (San Francisco, CA, USA, 2014), pp. 258-259. https://doi.org/10.1109/ISSCC.2014.6757425.

5. F. Golcuk, O.D. Gurbuz, G.M. Rebeiz, IEEE Trans. Microw. Theory Techn. 61(12), 4483 (2013). https://doi.org/10.1109/TMTT.2013.2287493.

6. Z. Hu, M. Kaynak, R. Han, IEEE J. Solid-State Circuits 53(5), 1313 (2018). https://doi.org/10.1109/ JSSC.2017.2786682. 
7. G. Guimaraes, P. Reynaert, in Proc. IEEE Int. Solid-State Circ. Conf. (San Francisco, CA, USA, 2020). https://doi.org/10.1109/ISSCC19947.2020.9063037.

8. H. Saeidi, S. Venkatesh, C.R. Chappidi, T. Sharma, C. Zhu, K. Sengupta, in Proc. IEEE Int. Solid-State Circ. Conf. (San Francisco, CA, USA, 2020), pp. 256-258. https://doi.org/10.1109/ISSCC19947. 2020.9063076.

9. H. Jalili, O. Momeni, IEEE J. Solid-State Circuits 55(9), 2387 (2020). https://doi.org/10.1109/JSSC. 2020.2989897.

10. K. Guo, P. Reynaert, in Proc. IEEE Int. Solid-State Circ. Conf. (San Francisco, CA, USA, 2020), pp. 442-444. https://doi.org/10.1109/ISSCC19947.2020.9063139.

11. R. Jain, P. Hillger, J. Grzyb, U.R. Pfeiffer, in Proc. IEEE Int. Solid-State Circ. Conf. (San Francisco, CA, USA, 2020), pp. 440-442. https://doi.org/10.1109/ISSCC19947.2020.9063025.

12. R. Jain, P. Hillger, E. Ashna, J. Grzyb, U.R. Pfeiffer, IEEE J. Solid-State Circuits 55(12), 3281 (2020). . https://doi.org/10.1109/JSSC.2020.3018819.

13. U.R. Pfeiffer, Y. Zhao, J. Grzyb, R. Al Hadi, N. Sarmah, W. Förster, H. Rücker, B. Heinemann, IEEE J. Solid-State Circuits 49(12), 2938 (2014). https://doi.org/10.1109/JSSC.2014.2358570.

14. K. Kasagi, S. Suzuki, M. Asada, J. Appl. Phys. 125(15), 1 (2019). https://doi.org/10.1063/1.5051007.

15. N. Shimizu, K. Matsuyama, H. Uchida, (San Francisco, California, United States, 2015), pp. 1-8. https://doi.org/10.1117/12.2076095.

16. K.B. Cooper, R.J. Dengler, N. Llombart, B. Thomas, G. Chattopadhyay, P.H. Siegel, IEEE Trans. THz Sci. Technol. 1(1), 169 (2011). https://doi.org/10.1109/TTHZ.2011.2159556.

17. S. Islam, J. Stiens, G. Poesen, I. Jaeger, R. Vounckx, Act. Passiv. Electron. Compon. 2008, 1 (2008). https://doi.org/10.1155/2008/391745.

18. H. Frid, IEEE Antennas Wireleless Propag. Lett. 15, 1963 (2016). https://doi.org/10.1109/LAWP. 2016.2545858.

19. M. Born, E. Wolf, Principles of optics: electromagnetic theory of propagation, interference and diffraction of light (Amsterdam, Netherlands: Elsevier, 2013).

20. D.B. Rutledge, D.P. Neikirk, D.P. Kasilingam, Infrared and millimeter waves 10(2), 1 (1983).

21. T.H. Buttgenbach, IEEE Trans. Microw. Theory Techn. 41(10), 1750 (1993). https://doi.org/10.1109/ 22.247920.

22. D.F. Filipovic, S.S. Gearhart, G.M. Rebeiz, IEEE Trans. Microw. Theory Techn. 41(10), 1738 (1993). https://doi.org/10.1109/22.247919.

23. D.F. Filipovic, G.P. Gauthier, S. Raman, G.M. Rebeiz, IEEE Trans. Antennas Propag. 45(5), 760 (1997). https://doi.org/10.1109/8.575618.

24. A.V. Boriskin, R. Sauleau, A.I. Nosich, IEEE Trans. Antennas Propag. 57(7), 2193 (2009). https:// doi.org/10.1109/TAP.2009.2021979.

25. A.V. Boriskin, R. Sauleau, JOSA A 27(11), 2442 (2010). https://doi.org/10.1364/JOSAA.27.002442.

26. A. Karttunen, J. Ala-Laurinaho, R. Sauleau, A.V. Räisänen, in Proc. Eur. Conf. Antennas Propag. (Barcelona, Spain, 2010), pp. 1-5.

27. S. Preu, C. Müller-Landau, S. Malzer, G.H. Doehler, H. Lu, A.C. Gossard, D. Segovia-Vargas, A. Rivera-Lavado, L.E. Garcia-Muñoz, IEEE Trans. Antennas Propag. 65(7), 3474 (2017). https://doi. org/10.1109/TAP.2017.2700039.

28. R. Al Hadi, et al., IEEE J. Solid-State Circuits 47(12), 2999 (2012). https://doi.org/10.1109/JSSC. 2012.2217851.

29. R. Zatta, R. Jain, D. Headland, U.R. Pfeiffer, in Proc. Int. Conf. Infrared Millim. Terahertz Waves (Paris, France, 2019), pp. 1-2. https://doi.org/10.1109/IRMMW-THz.2019.8874075.

30. D. Headland, R. Zatta, U. Pfeiffer, in Proc. Int. Conf. Infrared Millim. Terahertz Waves (Nagoya, Japan, 2018), pp. 1-2. https://doi.org/10.1109/IRMMW-THz.2018.8509901.

31. D. Liu, U. Pfeiffer, J. Grzyb, B. Gaucher, Advanced millimeter-wave technologies: antennas, packaging and circuits (John Wiley \& Sons, 2009).

32. J. Grzyb, H. Sherry, Y. Zhao, R. Hadi, A. Cathelin, A. Kaiser, U. Pfeiffer, Proc. SPIE 8362, 10 (2012). https://doi.org/10.1117/12.919218.

33. Y. Nishida, N. Nishigami, S. Diebold, J. Kim, M. Fujita, T. Nagatsuma, Sci. Rep. 9, art. no. 18125, 1 (2019). https://doi.org/10.1038/s41598-019-54627-8.

34. L. Liebermeister, S. Nellen, R. Kohlhaas, S. Breuer, M. Schell, B. Globisch, J. Infrared Millim. Terahertz Waves 40(3), 288 (2019). https://doi.org/10.1007/s10762-018-0563-6.

35. S. Preu, G. Döhler, S. Malzer, L. Wang, A. Gossard, J. Appl. Phys. 109(6), 4 (2011). https://doi.org/ $10.1063 / 1.3552291$. 
36. M. Andree, J. Grzyb, R. Jain, B. Heinemann, U.R. Pfeiffer, in Proc. IEEE MTT-S Int. Microw. Symp. (Boston, MA, USA, 2019), pp. 500-503. https://doi.org/10.1109/MWSYM.2019.8700871.

37. R. Jain, J. Grzyb, U.R. Pfeiffer, IEEE Trans. THz Sci. Technol. 6(5), 649 (2016). https://doi.org/10. 1109/TTHZ.2016.2584861.

38. R. Jain, F. Landskron, J. Grzyb, U.R. Pfeiffer, in Proc. Int. Conf. Infrared Millim. Terahertz Waves.

39. R. Jain, M. Schellenbeck, J. Grzyb, U.R. Pfeiffer, in Proc. Int. Conf. Infrared Millim. Terahertz. Waves (Cancun, Mexico, 2017). https://doi.org/10.1109/IRMMW-THz.2017.8067173.

40. D. Headland, R. Zatta, P. Hillger, U.R. Pfeiffer, IEEE Trans. THz Sci. Technol. 10(5), 513 (2020). https://doi.org/10.1109/TTHZ.2020.3004516.

41. R. Zatta, U.R. Pfeiffer, IEEE Trans. THz Sci. Technol. (2021). https://doi.org/10.1109/TTHZ.2021. 3088278 .

42. D. Headland, P. Hillger, R. Zatta, U. Pfeiffer, in Proc. Int. Conf. Infrared Millim. Terahertz Waves (Nagoya, Japan, 2018), pp. 1-2. https://doi.org/10.1109/IRMMW-THz.2018.8510246.

43. R. Zatta, V. Jagtap, J. Grzyb, U.R. Pfeiffer, IEEE Trans. THz Sci. Technol. (2021). https://doi.org/10. 1109/TTHZ.2021.3088286.

44. V.S. Jagtap, R. Zatta, J. Grzyb, U.R. Pfeiffer, in Proc. Int. Conf. Infrared Millim. Terahertz Waves (Paris, France, 2019), pp. 1-2. https://doi.org/10.1109/IRMMW-THz.2019.8874478.

45. L.E. Marchese, M. Terroux, M. Bolduc, M. Cantin, O. Martin, N. Desnoyers, A. Bergeron, in Proc. Int. Conf. Infrared Millim. Terahertz. Waves (Tucson, AZ, USA, 2014), pp. 1-2. https://doi.org/10. 1109/IRMMW-THz.2014.6956039.

46. D. Corcos, N. Kaminski, E. Shumaker, O. Markish, D. Elad, T. Morf, U. Drechsler, W.T. Silatsa Saha, L. Kull, K. Wood, U.R. Pfeiffer, J. Grzyb, IEEE Trans. THz Sci. Technol. 5(6), 902 (2015). https:// doi.org/10.1109/TTHZ.2015.2466470.

47. R. Al Hadi, J. Grzyb, B. Heinemann, U.R. Pfeiffer, IEEE J. Solid-State Circuits 48(9), 2002 (2013). https://doi.org/10.1109/JSSC.2013.2265493.

48. R. Han, Y. Zhang, Y. Kim, D.Y. Kim, H. Shichijo, E. Afshari, K.K. O, IEEE J. Solid-State Circuits 48(10), 2296 (2013). https://doi.org/10.1109/JSSC.2013.2269856.

49. R. Zatta, R. Jain, J. Grzyb, U. Pfeiffer, Terahertz Sci. Techn.-Int. J. THz 11(4), 102 (2018). http:// www.tstnetwork.org/10.11906/TST.102-123.2018.12.10.

50. A. Rivera-Lavado, S. Preu, L.E. García-Muñoz, A. Generalov, J. Montero-de Paz, G. Döhler, D. Lioubtchenko, M. Méndez-Aller, F. Sedlmeir, M. Schneidereit, et al., IEEE Trans. Antennas Propag. 63(3), 882 (2015). https://doi.org/10.1109/TAP.2014.2387419.

Publisher's Note Springer Nature remains neutral with regard to jurisdictional claims in published maps and institutional affiliations. 